\title{
AN EFFECTIVE VERSION OF HALL'S THEOREM
}

\author{
HENRY A. KIERSTEAD
}

\begin{abstract}
Manaster and Rosenstein [1972] constructed a recursively bipartite highly recursive graph that satisfies Hall's condition for a bipartite graph to have a matching, but has no recursive matching. We discuss a natural extension of Hall's condition which assures that every such graph has a recursive matching.
\end{abstract}

A bipartite graph $G=(A, B, E)$ consists of a set of vertices partitioned into $A$ and $B$ and a set $E$ of edges such that $E \subset A \times B$. A matching from $A$ to $B$ is a function $m: A \rightarrow B$ such that for all $a \in A,(a, m(a)) \in E$. For any $X \subset A$ let $N_{G}(X)=$ $\{b \in B$ : for some $a \in A,(a, b) \in E\}$ and $s_{G}(X)=\left|N_{G}(X)\right|-|X|$. When $G$ is clear from the context we shall drop the subscript.

Phillip Hall proved the following

TheOREM 0 (P. HALl [1935]). If $G=(A, B, E)$ is a finite bipartite graph then there is a matching from $A$ into $B$ if for all $X \subset A, s(X) \geqslant 0$.

Marshall Hall provided the following extension of this theorem to infinite graphs. Let $X \subset \subset A$ denote that $X$ is a finite subset of $A$.

TheOREM 1 (M. HALl [1948]). If $G$ is a bipartite graph such that for every $a \in A$, $N(\{a\})$ is finite then $G$ has a matching from $A$ into $B$ iff for all $X \subset \subset A, s(X) \geqslant 0$.

We shall say that $G$ satisfies the Hall condition, denoted H.c., if for all $X \subset \subset A$, $s(X) \geqslant 0$. Theorem 1 is somewhat unsatisfying because, while it asserts the existence of a matching, it does not provide an algorithm for constructing that matching. In fact, in a sense made precise by the following theorem there may be no such algorithm.

THEOREM 2 (MANASTER AND ROSENSTEIN [1972]). There is a recursively bipartite, highly recursive graph $G=(A, B, E)$ which satisfies the H.c. but does not have a recursive matching.

Some explanation is in order. Roughly speaking, a graph $G$ is recursive if there are algorithms for computing both its vertex set and its edge set; $G$ is highly recursive if it is recursive, every vertex has finite degree and there is an algorithm for computing these degrees; $G$ is recursively bipartite if it is bipartite, say $G=(A, B, E)$, and there is an algorithm for determining membership in $A$ and $B$. Thus a locally finite

Received by the editors June 18, 1982.

1980 Mathematics Subject Classification. Primary 03D45.

' Supported in part by NSF grant ISP-80-11451.

(C) 1983 American Mathematical Society 0002-9939/82/0000-0995/\$02.00 
bipartite graph $G$ is a recursively bipartite, highly recursive graph if there are algorithms for determining all "local" information about $G$. A matching $m$ is recursive if there is an algorithm for computing it. For a more detailed and very readable account of recursive functions, the reader is referred to $\S 1$ of Manaster and Rosenstein [1972].

In this paper we provide a condition, similar in spirit to the H.c., which guarantees that a recursively bipartite, highly recursive graph $G=(A, B, E)$ will have a recursive matching from $A$ to $B$. We also show that this condition cannot be relaxed by constructing a recursively bipartite, highly recursive graph that satisfies "most" of the condition but does not have a recursive matching. This construction is similar to the construction in Manaster and Rosenstein [1973] but requires a different argument to show that it accomplishes its objective. Finally, we use our condition to derive a noneffective result for countable graphs with infinite degree. The reader is referred to Holz, Podewski, and Steffens [1979] for further results of this nature.

Definition. Let $G=(A, B, E)$ be a bipartite graph. $G$ satisfies the expanding Hall condition, denoted e.H.c. iff there exists a function $h: N \rightarrow N$ such that $h(0)=0$ and for all $X \subset \subset A, h(n) \leqslant|X|$ implies $n \leqslant s(X)$. $G$ satisfies the recursive expanding Hall condition, denoted r.e.H.c., iff $G$ satisfies the e.H.c. and there is a recursive function $h$ that witnesses this.

Notice that since $h(0)=0$, the e.H.c. implies the H.c.

THEOREM 3. If $G=(A, B, E)$ is a recursively bipartite highly recursive graph which satisfies the r.e.H.c., then $G$ has a recursive matching $m$ of $A$ into $B$.

Proof. Let $h$ witness the r.e.H.c. for $G$. Let $a_{0}$ be the first element of $A$. Form an induced subgraph $G_{0}=\left(A_{0}, B_{0}, E_{0}\right)$ of $G$ by taking $A_{0} \cup B_{0}$ to be the set of vertices a distance of at most $2 h(1)+1$ from $a_{0}$. This can be done effectively and $G_{0}$ is finite because $G$ is highly recursive. Using the finite version of Hall's Theorem, construct a matching $m_{0}$ of $A_{0}$ into $B_{0}$. Let $m\left(a_{0}\right)=m_{0}\left(a_{0}\right)=b_{0}$. It suffices to show that $G^{\prime}=G-\left\{a_{0}, b_{0}\right\}$ is still a recursively bipartite, highly recursive graph which satisfies the r.e.H.c., for then we can just iterate the above process. The first part is immediate, so we consider the r.e.H.c. We begin by showing that $G^{\prime}$ satisfies the H.c. Let $X \subset \subset A^{\prime}=A-\left\{a_{0}\right\}$. If $X \subset A_{0}$ then $s_{G^{\prime}}(X) \geqslant 0$ since $m_{0}$ is a matching of $A_{0}$ into $B_{0}$. If $b_{0} \notin N(X)$ then $s_{G^{\prime}}(X)=s_{G}(X) \geqslant 0$. Finally suppose that $\left(b_{0}, a_{1}\right) \in E$, $a_{1} \in X$ and there exists $a_{2} \in X-A_{0}$. Without loss of generality $X$ is connected. Thus by the choice of $G_{0}$ there is a path of length at least $2 h(1)+1$ from $a_{1}$ to $a_{2}$; so $|X| \geqslant h(1)+1>h(1)$ and $s_{G^{\prime}}(X) \geqslant s_{G}(X)-1 \geqslant 0$. Now let $h^{\prime}(n)=h(n+1)$ for all $n>0$. Clearly, if $|X| \geqslant h^{\prime}(n)$ then $|X| \geqslant h(n+1), s_{G}(X) \geqslant n+1$, and $s_{G^{\prime}}(X)$ $\geqslant n$.

The author thanks Alfred Manaster for his helpful suggestions for presenting the proof of Theorem 3. The r.e.H.c was first formulated by the author to prove the following result.

Corollary 4. Let $G=(A, B, E)$ be a highly recursive, bipartite graph such that every vertex in $A$ has degree greater than $n$ and every vertex in $B$ has degree less than or equal to $n$. Then $G$ has a recursive matching. 
Proof. First note that $G$ is recursively bipartite since we can decide membership in $A$ or $B$ via the recursive degree function. So by the theorem it suffices to find a recursive function $h$ that witnesses the r.e.H.c. for $G$. Using the fact that if $X \subset \subset A$ then $(n+1)|X| \leqslant n|N(X)|$, it is easy to check that $h(x)=n x$ is such a function.

For the proof of the next theorem we shall need to make use of the usual effective listing $\phi_{0}, \phi_{1}, \ldots$ of all algorithmically computable functions. We write $\phi_{e}^{k}(x)=y$ if the $e$ th algorithm stops after $k$ steps when started on $x$ and gives output $y ; \phi_{e}^{k}(x)$ is not defined if the $e$ th algorithm does not stop in $k$ steps when started on $y$. Again the reader is referred to $\S 1$ of Manaster and Rosenstein [1972] for more details.

THEOREM 5. There exists a highly recursive, recursively bipartite graph $G=$ ( $A, B, E)$ which satisfies the e.H.c. but does not have a recursive matching of $A$ into $B$.

Proof. Fix a recursive partition $\left\{P_{e}: e \in N\right\}$ of $N$ into infinitely many infinite recursive pieces. Our graph $G$ will be the disjoint union of infinitely many independent graphs $G_{e}=\left(A_{e}, B_{e}, E_{e}\right), e \in N$, which satisfy the following conditions. We denote $A_{e} \cup B_{e}$ by $V_{e}$.

(0) $A_{e}, B_{e} \subset P_{e}$;

(1) $\phi_{e}$ is not a matching of $A_{e}$ into $B_{e}$;

(2) for all $X \subset \subset A_{e}$, if $\left|V_{e}\right|=\infty$ then $|X| \leqslant s(X)$;

(3) for all $X \subset \subset A_{e}, \min (e,|X|) \leqslant s(X)$.

Suppose that we have accomplished this. Since any matching of $A$ into $B$ is a matching of $A \cap X$ into $B \cap X$ for any component $X$, by (1) $G$ does not have a recursive matching from $A$ to $B$. To see that $G$ satisfies the e.H.c., we fix an arbitrary $m$ and determine $n$ such that for all $X \subset \subset A, n \leqslant|X|$ implies that $m \leqslant s(X)$. In light of Theorem 1, we certainly cannot do this effectively. Let $D=\{e: e<m$ and $\left.\left|V_{e}\right|<\infty\right\}, d=\left|\cup_{i \in D} V_{i}\right|$, and $n=m+d$. If $X \subset \subset A$ and $n \leqslant|X|$, then,

$$
\begin{aligned}
s(X) & =\sum_{i \in N} s\left(X \cap V_{i}\right) \\
& \geqslant \sum_{\substack{i<m \\
i \notin D}} s\left(X \cap V_{i}\right)+\sum_{i \geqslant m} s\left(X \cap V_{i}\right) \\
& \geqslant \sum_{\substack{i<m \\
i \notin D}}\left|X \cap V_{i}\right|+\sum_{i \geqslant m} \min \left(m,\left|X \cap V_{i}\right|\right) \\
& \geqslant \min (m,|X|-d) \geqslant m .
\end{aligned}
$$

In order to describe the construction of $G_{e}$ we must introduce some notation. Let $F(h, e)=\left(A_{F}, B_{F}, E_{F}\right)$ be the forest consisting of $3 e$ trees $T_{i}(h), i<3 e$, of the form in the diagram where each $S(h, i, j)$ is a full binary tree of height $2 h+1$ whose root is $s_{i}^{j}$. Let $L(k, i, j)$ be the vertices of $S(h, i, j)$ at level $k$. (The root $s_{i}^{j}$ is at level 0 .) Let

$$
A_{f}=\bigcup_{\substack{k \leq h \\ i<3 e \\ j<3}} L(2 k, i, j) \cup\left\{r_{i}: i<e\right\}
$$


and

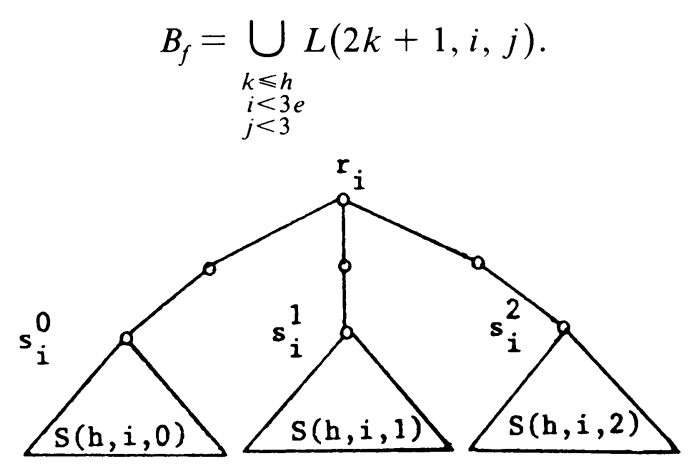

We shall construct $G_{e}$ in stages. $G_{e}=\left(A_{e}^{h}, B_{e}^{h}, E_{e}^{h}\right)$ will be the part of $G_{e}$ constructed by the end of the $h$ th stage. If the $n$th element of $P_{e}$ is a vertex in $V_{e}$, it will be in $V_{e}^{n}=A_{e}^{n} \cup B_{e}^{n}, G_{e}^{h}$ will be an induced subgraph of $G_{e}$, and no vertex introduced at stage $h+1$ will be adjacent to any vertex introduced before stage $h$. Assuming that the construction of the $G_{e}^{h}$ 's will be effective, the first two conditions assure that $G_{e}$ is recursive and recursively bipartite and this together with the last condition assures that $G_{e}$ is highly recursive. Let $G_{e}^{0}$ be isomorphic to $F(0, e)$ where $V_{e}^{0}$ consists of the first $3 e \cdot 13$ elements of $P_{e}$ and the assignment of vertices of $V_{e}^{0}$ to vertices of $F(0, e)$ is made in some reasonable fashion. Now proceeding inductively, suppose that we have defined $G_{e}^{h}$.

Case 1. If $\phi_{e}^{h}$ is not a matching of $\left\{r_{0}, \ldots, r_{3 e-1}\right\}$ into $B_{e}$, let $C_{e}^{h+1}$ be an extension of $G_{e}^{h}$ to an isomorphic copy of $F(h+1, e)$ formed in some reasonable way from the next $9 e\left(2^{2 h}+2^{2 h+1}\right)$ elements of $P_{e}$.

Case 2. Suppose $h$ is the first natural number such that $\phi_{e}^{h}$ is a matching from $\left\{r_{0}, \ldots, r_{3 e-1}\right\}$ into $B_{e}$. For each $i<3 e-1$ let $j(i)$ be such that $\left(\phi_{e}^{h}\left(r_{i}\right), s_{s}^{j(i)}\right) \in E_{e}$. Let $I$ be the next $e 2^{2(h+1)}$ elements in $P_{e}$. Now form $G_{e}^{h+1}$ by making each vertex of $I$ adjacent to each vertex of $\bigcup_{i<e} L(2 h+1, i, j(i))$ and putting the vertices of $I$ into $A_{e}$.

Case 3. Otherwise let $G_{e}^{h+1}=G_{e}^{h}$.

Now we check (1)-(3). For (1), notice that if we never enter Case 2 then $\phi_{e}$ is not a matching of $A_{e}$ into $B_{e}$. So suppose at stage $h+1$ we are in Case 2. Consider the subgraph $H$ of $G_{e}$ induced by $I=\cup_{i<3 e} S(h, i, j(i))$. By the choice of $j(i)$, if $\phi_{e}$ is a matching of $A_{e}$ into $B_{e}$, then $\phi_{e}$ is a matching of $H \cap A_{e}$ into $H \cap B_{e}$. But this is impossible since

$$
\left|H \cap A_{e}\right|=3 e \sum_{i=0}^{h} 2^{2 i}+e 2^{2(h+1)}=e \cdot 2 \cdot 4^{h+1}-e
$$

and

$$
\left|H \cap B_{e}\right|=3 e \sum_{i=0}^{h} 2^{2 i+1}=e \cdot 2 \cdot 4^{h+1}-2 e .
$$

To prove (2), we remark that if $\left|V_{e}\right|=\infty$ then $G_{e}$ is a forest and every vertex in $A_{e}$ has degree 3. Thus for $X \subset \subset A_{e}, s(X) \geqslant|X|$. Finally suppose $X \subset \subset A_{e}$ where 
$\left|V_{e}\right|<\infty$. Since $\left|V_{e}\right|<\infty$ we reached Case 2 in the construction of $G_{e}$ at some stage $h$. Notice that if $X \cap I=\varnothing$ then just as for (2), $S(X) \geqslant|X|$. So suppose $X \cap I \neq$ $\varnothing$. Let $H^{\prime}$ be the graph induced by the vertices of $H$ together with $\left\{\phi_{e}\left(r_{i}\right): i<3 e\right\}$. Let $F$ be $G_{e}-H^{\prime}$. Then $F$ is a forest with roots $r_{i}$, for $i<3 e$, and every vertex in $F \cap X$ has degree 3 or is a root of degree 2. Thus $s_{F}(F \cap X)$ is greater than or equal to $|X|$. So it suffices to show that $s\left(H^{\prime} \cap X\right)$ is at least $\min (e,|X|)$. Each $\phi_{e}\left(r_{i}\right)$ is adjacent only to $s_{i}^{j(i)}$ in $H^{\prime}$. Thus $s\left(H^{\prime} \cap X\right) \geqslant s(U \cap X)$ where $U$ is the set of vertices of $H$ with the roots $s_{i}^{j(i)}$ removed. Since each element of $L(2 h+1, i, j(i))$ is adjacent to each element of $I$, if $|U \cap X|<e$ then

$$
s^{\prime}(U \cap X) \geqslant e \cdot 2^{2(h+1)}-e \geqslant 3 e \geqslant|U \cap X| .
$$

Now suppose $|U \cap X| \geqslant e$. Thus it suffices to show that $e \leqslant s(U \cap X)$. We can count $N(U \cap X)$ by counting $L(2 h+1, i, j(i))$ together with the elements of $L(2 k-1, i, j(i))$ that are adjacent to elements of $L(2 k, i, j(i)) \cap X$ for $0<k \leqslant h$. Since elements of $L(2 k-1, i, j(i))$ are adjacent to only two elements of $L(2 k, i, j(i))$ and every element of $L(2 k, i, j(i))$ is adjacent to some element of $L(2 k-1, i, j(i))$,

$$
N(U \cap X) \geqslant 3 e^{2 h+1}+\frac{1}{2}|(U-I) \cap X|
$$

and

$$
\begin{aligned}
s(U \cap X) & \geqslant 3 e 2^{2 h+1}-\frac{1}{2}|(U-I) \cap X|-|I \cap X| \\
& \geqslant 6 e 4^{h}-\frac{1}{2} \cdot 3 e \sum_{i=1}^{h} 4^{i}-4 e 4^{h} \geqslant 2 e .
\end{aligned}
$$

THEOREM 6. Let $G=(A, B, E)$ be a countable bipartite graph. If $G$ satisfies the e.H.c. then $G$ has a matching from $A$ into $B$.

Proof. Let $a_{0}$ be the first element of $A$. As in the proof of Theorem 3, it suffices to match $a_{0}$ to $b_{0} \in B$ in such a manner that $G^{\prime}=G-\left\{a_{0}, b_{0}\right\}$ satisfies the e.H.c. As before the only difficulty is in checking that $G^{\prime}$ satisfies the H.c. We must consider two cases: (1) $\delta(a)<\infty$ and (2) $\delta(a)=\infty$. For (1), let $A_{f}=\{a \in A$ : $\delta(a)<\infty\}$ and $G_{f}$ be the graph induced by $A_{f} \cup N\left(A_{f}\right)$. By Theorem $1, G_{f}$ has a matching $m$ from $A$ to $B$. Clearly, letting $b_{0}=m\left(a_{0}\right)$ endows $G^{\prime}$ with the H.c. For case (2), let $A_{0}$ be the maximum subset of $A$ such that $s\left(A_{0}\right)=0$. The e.H.c. assures that $A_{0}$ exists and is finite. Let $b_{0}$ be the least element of $N\left(\left\{a_{0}\right\}\right)-N\left(A_{0}\right)$. Again it is easy to check that $G^{\prime}$ satisfies the H.c.

\section{REFERENCES}

M. Hall (1948) Distinct representatives of subsets, Bull. Amer. Math. Soc. 54, 922-926.

P. Hall (1935) On representatives of subsets, J. London Math. Soc. 10, 26-30.

M. Holz, K.-P. Podewski and K. Steffens (1979) Hall families and the marriage problem, J. Combinatorial Theory Ser. A 27, 161-180.

A. Manaster and J. Rosenstein (1972) Effective matchmaking (recursion theoretic aspects of a theorem of Philip Hall), Proc. London Math. Soc. 25, 615-654. (1973) Effective matchmaking and k-chromatic graphs, Proc. Amer. Math. Soc. 39, 371-378.

Department of Mathematics and Statistics, University of South Carolina, Columbia, South CAROLINA 29208 